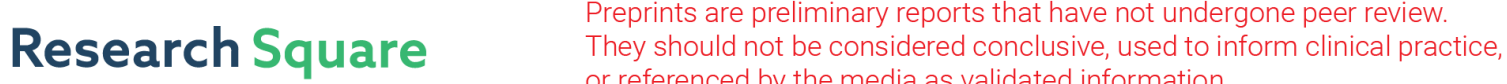 or referenced by the media as validated information. \\ Calculation of the Fine Structure Constant derived from Principle Theory.
}

Manfred Geilhaupt ( $\square$ manfred.geilhaupt@hsnr.de)

University of Applied Sciences Niederrhein

Research Article

Keywords: Fine Structure Constant, General Relativity, Thermodynamics

Posted Date: July 14th, 2021

DOl: https://doi.org/10.21203/rs.3.rs-696488/v1

License: (c) (i) This work is licensed under a Creative Commons Attribution 4.0 International License.

Read Full License 


\title{
Calculation of the Fine Structure Constant derived from Principle Theory.
}

\author{
Manfred Geilhaupt \\ Hessenfeld 10, 41844 Wegberg, Germany
}

\begin{abstract}
Recently we have presented in a paper that the combination of two Principle Theories, General Relativity (GR) and Thermodynamics (TD), is able to derive the restmass ( $m$ ) of an electron which (surprisingly) depends on the Sommerfeld Fine Structure Constant (FSC). In this paper we present a completed calculation of the FSC number $(1 / \alpha=137.035999024(9))$ from a GR+TD point of view.
\end{abstract}

Since the introduction of the Higgs mechanism, the Standard Model (SM) presents an explanation of (inertial) mass contribution from interaction with the Higgs-Field ${ }^{[1]}$ but the FSC is not involved. In our two preprints ${ }^{[2]}$ we showed independently form the Higgs-Model that elementary particles restmass depends on the FSC number ${ }^{[2.1]}$.

Nevertheless we have to take into account a Higgs contribution on restmass due to permanent interaction with sub-quantized W-Bosons, Z-Boson, and Higgs-Boson as the (SM) requires. The electron self-energy is based on an interaction not only with "itself" due to 5 inertial ("internal") forces ${ }^{[2.1]}$ but also interacts with the Higgs-Field ${ }^{[1]}$ and with an electroweak-field ${ }^{[1]}$. Let us assume an absent external electromagnetic field and its possible interaction with photons ${ }^{[3]}$.

From GR+TD basics ${ }^{[2]}$, Einstein's equation of motion ${ }^{[3]}$, and the following differential equation 1.1 gives $\mathrm{m}(\mathrm{t})^{[2.1]}$ while introducing $\mathrm{r}(\mathrm{t})^{[2.1]}$ (mass generating function).

$1.1 \quad m(t) \cdot c^{2}=-\frac{2}{\kappa(t)} \cdot \frac{\partial^{2} r(t)}{\partial t^{2}} \cdot 4 \pi r^{2}(t)$

Finally we have from "common" mathematics the "effective" mass value $\mathrm{m}_{\mathrm{e}}{ }^{[2.1]}$

$1.1 .1 m_{e}=\sqrt{h \cdot c / G_{0} \cdot 2 \alpha / 4 \pi \cdot 24 / N_{e}^{2}}$

to be compared with the experimental value. So the very new result from 1.1.1 is: "Mass depends on alpha".

In so far as GR+TD must include permanent interaction with Quantum-Fields as there are: Gravity-, Higgs- and Weak-em-Field then we must have a focus on alpha while including both $\mathrm{SM}^{[1]}$ and GR+TD ${ }^{[2]}$ theoretical and experimental facts ${ }^{[1,2,3]}$. (We leave out here the 
discussion about the second parameter $\mathrm{N}$, initial Quantum-Number, explaining the quantisation of elementary particles mass ${ }^{[2.1]}$.)

The GR+TD result of the FSC is ${ }^{[2]}$ :

$$
\alpha=\frac{1}{g_{44}} \frac{1}{\left(\beta_{\mathrm{int}}\right)^{2}}(3 / 4) \cdot\left(1 / \beta_{\mathrm{int}} \cdot \ln (1 / 3)+1\right)^{2}
$$

Beta and g44 are two (formally well know) free SR and GR parameters. Let us first assume the most simple aspects g44=beta=1 to come up with a first zero approximation of alpha

$$
\alpha_{0}=(3 / 4) \cdot(\ln (1 / 3)+1)^{2}=1 / 137.112377003
$$

The next step is a hypothesis that the inertial restmass (GR+TD based 5-force ${ }^{[2.1]}$ action) is accompanied by a limiting velocity $v_{0}=\alpha_{0} * c<c$ because we are discussing non zero restmass.

Notice: Sommerfeld mentioned $v_{e}=\alpha^{*} c$ is the orbit velocity of the "point-like" electron in a Hydrogen atom first orbit. In our approach $v_{e}=\alpha^{*} c$ might be the initial action velocity of a non-point like Electron with centre at rest. Our hypothesis respects that initial and orbit action of an electron matches in so far if both internal and external periodic action is completed at the same time.

Now assume still g44=1 and we have a next absolute and constant (Einstein SR based) alpha number while introducing the hypothesis beta $0=\operatorname{sqrt}\left(1-\left(v_{0} / c\right)^{2}\right)$ from above.

$$
\begin{aligned}
& \beta_{0}=\sqrt{1-\left(\alpha_{0}\right)^{2}}=0.999973403624 \\
& \alpha=\frac{1}{g_{44}} \cdot \frac{1}{\left(\beta_{0}\right)^{2}}(3 / 4) \cdot\left(1 / \beta_{0} \cdot \ln (1 / 3)+1\right)^{2}=1 / 137.023868391
\end{aligned}
$$

This alpha-value 2.1.3 can be completed by 944 from theory. Then alpha might be compared with the experimental values given from Parker ${ }^{[4]}$ and Morel ${ }^{[5]}$. Both experiments present a high precision alpha-number from different experiments. If g44 would be different in number the tension between Parker (137.035999046) and Morel (137.035999206) might be understood from a general theoretical point of view. From the SM point of view alpha must be a constant. In so far the two experiments reveal a problem pointing beyond the SM.

To come up with a "running" alpha from GR+TD, we accept the following internal and external action contribution on the metric number $\mathrm{g} 44$. These contributions are:

- "Internal" Yukawa and the W, Z, Higgs-Boson contributions.

- "External" Gravity Field contributions from earth and sun on earth.

Definition:

external: "convex GR-Schwarzschild metric contribution (negative sign)"

internal: "concave GR+SM-action contribution (positive sign)". 
So at least there are 4 contributions:

$$
2.2 g_{44}=1+\left(g^{2} e^{-2 m * c * \lambda / \hbar}+\Phi_{W Z H}\right)-\frac{2 G M_{E}}{c^{2} R_{E}}-\frac{2 G M_{S}}{c^{2} R_{S E}}
$$

Constants (Codata) used for the calculation of the metric g44 number.

$\begin{array}{lll}\text { h } & 6,626070150 E-34 ~ J s \\ \text { c } & 2,997924580 E+08 \mathrm{~m} / \mathrm{s} \\ \text { e } & 1,602176634 \mathrm{E}-19 \mathrm{C} \\ \text { RSonne } & 6,695700000 \mathrm{E}+08 \mathrm{~m} \\ \text { MSonne } & 1,988920000 \mathrm{E}+30 \mathrm{~kg} \\ \text { PI } & 3,141592654 \mathrm{E}+00 \\ \mathrm{G} & 6,674300000 \mathrm{E}-11 \mathrm{~m}^{\wedge} 3 / \mathrm{kg} / \mathrm{s}^{\wedge} 2 \\ \text { MErde } & 5,972200000 \mathrm{E}+24 \mathrm{~kg} \\ \text { RErde } & 6,371000000 \mathrm{E}+06 \mathrm{~m} \\ \text { RSoEr } & 1,495936290 \mathrm{E}+11 \mathrm{~m} \\ \text { RMond } & 1,738000000 \mathrm{E}+06 \mathrm{~m} \\ \text { MMond } & 7,349000000 \mathrm{E}+22 \mathrm{~kg} \\ \text { RMoEr } & 3,844000000 \mathrm{E}+08 \mathrm{~m} \\ & & \\ \text { me } & 5,109989500 \mathrm{E}+05 \mathrm{eV} \\ \mathrm{mW} & 8,037900000 \mathrm{E}+10 \mathrm{eV} \\ \mathrm{mZ} & 9,118700000 \mathrm{E}+10 \mathrm{eV} \\ \mathrm{mH} & 1,219000000 \mathrm{E}+11 \mathrm{eV} \\ \text { epsO } & 8,854187183 \mathrm{E}-12 \mathrm{As} / \mathrm{Vm}\end{array}$

We start with the internal Yukawa metric number contribution $\Phi_{\mathrm{YK}}$. The degeneration number is $\mathrm{g}=5$ due to five GR-internal force contributions ${ }^{[2.1]}$.

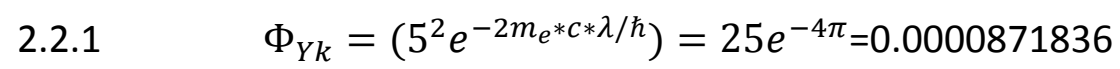

So $\Phi_{Y K}$ must be a constant.

Notice:

The internal Yukawa number from 2.2.1 is based on using the relation $\left(4 \pi^{*} r G\right) * m_{e}{ }^{*} c=h$, defining the Compton wave $\lambda=\left(4 \pi^{*} r G\right)$ that way. Here $r G$ is the effective value form solution $r(t)^{[2.1]}$ which leads to $m(t){ }^{[2.1]}$ and then to the effective value $m_{e}{ }^{[2.1]}$ in accordance with Einstein's: $m_{e}{ }^{*} c^{2}=h * c / \lambda$ equation. Thus we can conclude theoretically: The Compton wavelength hypothesis now is "explained" by GR+TD due to the rG-result ${ }^{[2.1]}$.

Next we discuss the SM metric contributions $\Phi_{\mathrm{WZH}}$. The three internal metric contributions are from $2 \mathrm{~W}$-Bosons, Z-Boson and Higgs-Boson. $\left(\Phi_{\mathrm{WZH}}\right.$ is my hypothesis and thus new in GRphysics but necessary to respect the Standard Model (SM) facts to be combined with GR+TD.)

2.2.2 $\Phi_{W Z H}=(2 m W+m Z+m H) \cdot c^{2} /\left(m_{e} \cdot c^{2}\right)=0.0000013669 \underline{10}$ 
Notice: Without the "potential energy of Bosons divided by the electron potential selfenergy" the alpha number is incomplete and would not respect the Higgs-Model contribution on restmass.

From 1.1.1 we know all Boson mass values must depend on the FSC number alpha as well. The same to the mass of the electron, so here alpha cancel at least within formula 2.2.2 (we use experimental mass values).

In so far as the experiments are executed on earth surface we discuss the earth metric influence. We start with the Schwarzschild metric contribution $\phi_{E}$ (earth mass $M_{E}$ and earth Radius RE). Here in the form of "potential energy divided by potential self-energy of the electron" (energy based definition).

$$
\varphi_{E}=-\frac{2 G M_{E} \cdot m_{e}}{R_{E} \cdot m_{e} \cdot c^{2}}=1.392 \underline{27} * 10^{-9}
$$

Restmass $m_{e}$ can be cancelled to get the pure Schwarzschild metric formula ${ }^{[3]}$.

The next contribution is the external Schwarzschild sun contribution $\phi_{S E}$ at earth $\left(R_{E}\right)$ surface while introducing the sun-mass $\left(M_{S}\right)$ and mean-distance $\left(R_{S E}=1.496 * 10^{11} \mathrm{~m}\right)$ value from experiment $\left(1.471 * 10^{11} \mathrm{~m}\right.$ (January) up to $1.521 * 10^{11} \mathrm{~m}$ (July)).

$$
\varphi_{S E}=-\frac{2 G M_{S} \cdot m_{e}}{R_{S E} \cdot m_{e} \cdot c^{2}}=1.974 \underline{78} * 10^{-8}
$$

As we can see, the sun on earth contribution is one order more in size than the earth contribution on the metric number itself. The main contribution is from 2.2.4 (uncertainty $12^{\text {th }}$ figure) and from 2.2.3 (uncertainty $14^{\text {th }}$ figure) less influence.

The external Schwarzschild moon contribution $\phi_{M}$ at the moon-surface while introducing the mass and distance values respectively is given in the next formula.

$$
\varphi_{M}=-\frac{2 G M_{M} \cdot m_{e}}{R_{M} \cdot m_{e} \cdot c^{2}}=6.280 \underline{22} * 10^{-11}
$$

The Schwarzschild sun on moon contribution $\phi_{S M}$ is not much different to the sun on earth contribution because of the nearly same distance.

$$
\varphi_{S M}=-\frac{2 G M_{M} \cdot m_{e}}{R_{S M} \cdot m_{e} \cdot c^{2}}=1.974 \underline{72} * 10^{-8}
$$

Conclusion: The alpha number must be different on earth and on moon. 
Table 1 Calculation Overview: GR+TD earth-FSC-calculation based on $\mathrm{g} 44=1+$ contributions from 2.2.1-2.2.4

\begin{tabular}{|c|c|c|c|c|c|}
\hline & $\Phi(\mathrm{YK})$ & $\Phi(\mathrm{WZH})$ & $\phi$ (Earth) & $\phi($ Sun-Earth) & $1 / \alpha$ \\
\hline 1 & 0 & 0 & 0 & 0 & 137.023868391 \\
\hline 1 & 0.000087183559 & 0 & 0 & 0 & 137.035814620 \\
\hline 1 & 0.00008718356 & $1.3669 \underline{1} * 10^{-6}$ & 0 & 0 & 137.036001919 \\
\hline 1 & 0.00008718356 & $1.3669101 * 10^{-6}$ & $-1.39227^{*} * 10^{-9}$ & 0 & 137.036001729 \\
\hline \multirow[t]{3}{*}{1} & 0.00008718356 & $1.3669101 * 10^{-6}$ & $-1.392269 * 10^{-9}$ & $-1.974 \underline{8}^{*} 10^{-8}$ & $137.03599902 \underline{4}$ \\
\hline & & January & Maximum $\mathrm{R}_{\mathrm{SE}}$ & $1.521 * 10^{+11} \mathrm{~m}$ & $137.03599897 \underline{8}(-46)$ \\
\hline & & July & Minimum $\mathrm{R}_{\mathrm{SE}}$ & $1.471 * 10^{+11} \mathrm{~m}$ & $137.03599906 \underline{9}(+45)$ \\
\hline
\end{tabular}

The alpha number (last column table 1 ) is completed step by step due to the metric contributions from (2.2) introduced into (2.1.3).

The last word has the experiment: In 2026 NASA and ESA are planning atomic interferometry experiments on space lab (private information).

The g44-metric (on earth experiments) includes three constant contributions (Yukawa, QED and Earth) and one non constant contribution due to parameter $R_{S E}$ (the sun-earth distance in January and July differs significantly). The change in alpha is shown in table 1 . It is useful to repeat the Parker and Morel experiments in January and July at the same time.

Experiment meets Theory

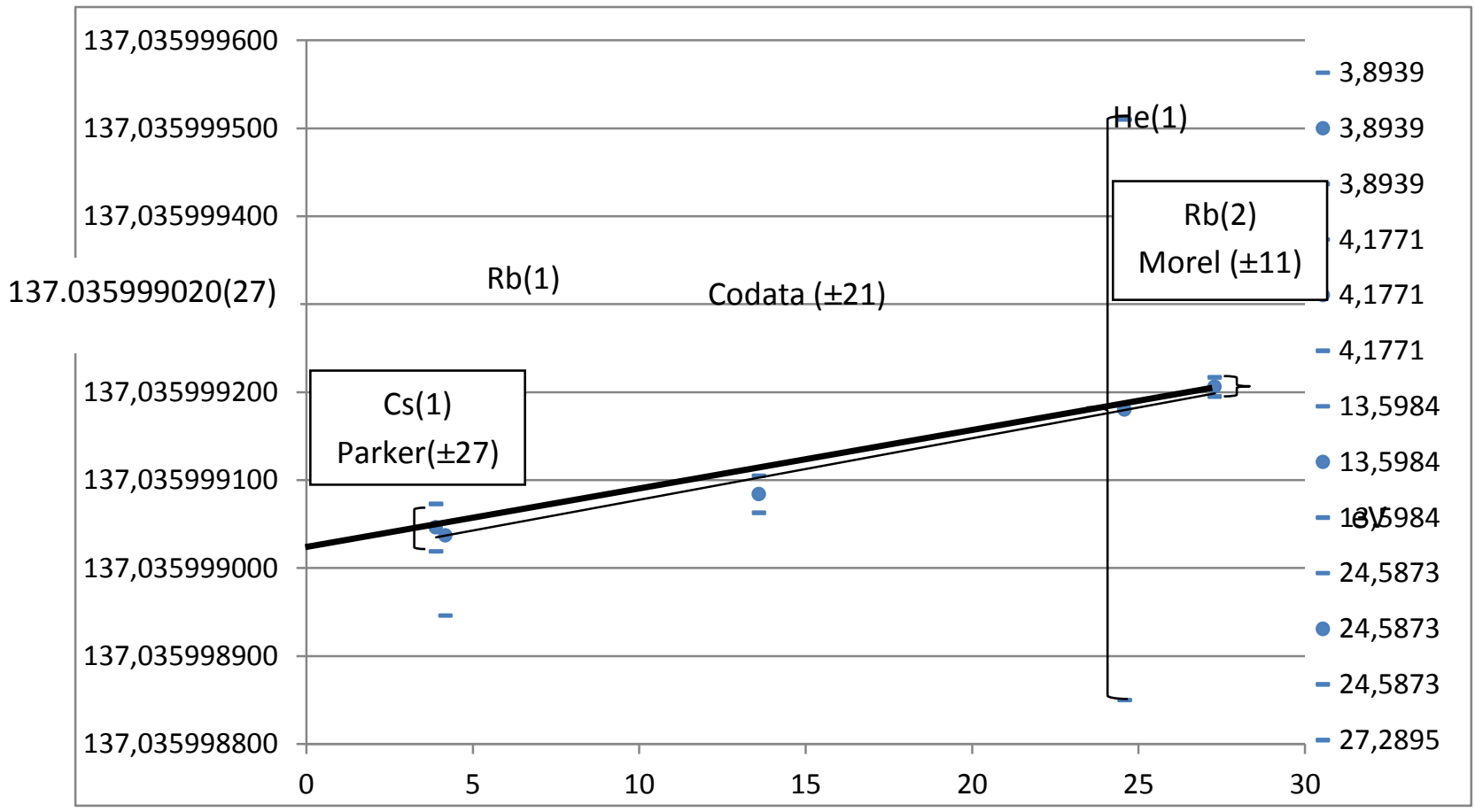

Figure 1 Experimental data (blue buttons, with blue error bars) indicate a linear dependence of the FSC from the atoms ionisation energy (x-axis). (Bold line, linear fit only from Morel and Parker) 
The calculation of 1/alpha $=137.035999024(9)$ from GR+TD (table 1 ) and the estimation of $1 /$ alpha(0eV)=137.035999020(27) (figure 1) ${ }^{[2.2]}$ are very close.

Table 2 Changing $R_{S E}$ (Minimum to Maximum) we estimate and predict significant differences in 1/alpha "between January and July".

\begin{tabular}{|l|l|l|l|l|}
\hline & Theory & Parker(Cs) & Morel(Rb) & Fit alpha(OeV) \\
\hline January(+46) & $137.03599907 \underline{\underline{2}}$ & 137.035999092 & 137.035999252 & 137.035999066 \\
\hline & $\mathbf{1 3 7 . 0 3 5 9 9 9 0 2 \underline { 4 }}$ & $\mathbf{1 3 7 . 0 3 5 9 9 9 0 4 6 ( 2 7 )}$ & $\mathbf{1 3 7 . 0 3 5 9 9 9 2 0 6 ( 1 1 )}$ & $\mathbf{1 3 7 . 0 3 5 9 9 9 0 2 0 ( 2 7 )}$ \\
\hline July(-46) & $137.03599897 \underline{8}$ & 137.035999000 & 137.035999160 & 137.035998982 \\
\hline & & & & \\
\hline
\end{tabular}

Conclusion: Both experiments ${ }^{[4,5]}$ repeated on earth at July and January at the same time should show a significant difference to prove or disprove our theoretical point of view concerning the FSC depends on g44 metric number. 
Suggesting further experiments

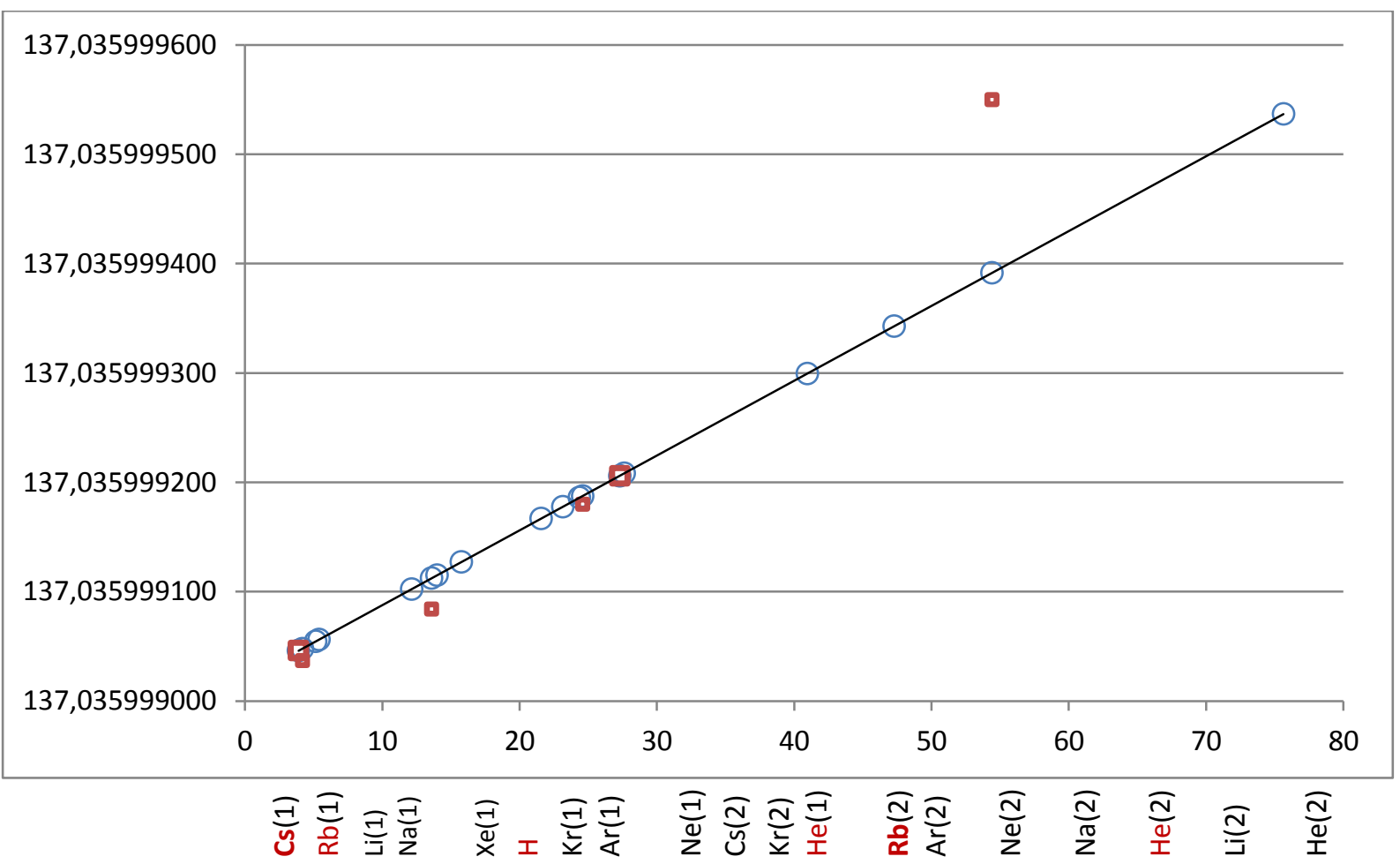

Figure 2 Theoretical predictions (from fit red line figure 1) of the FSC number (blue circle) depending on the $1^{\text {st }}$ and $2^{\text {nd }}$ ionisation energy of the emitting electron from different atoms including six experimental results (red square) presented in table 3

Table 3 Experimental results introduced into figure 2 (red square buttons)

\begin{tabular}{|l|l|l|l|l|}
\hline & eV & $\begin{array}{l}\text { From fit (red } \\
\text { line) }\end{array}$ & Experiment & \\
\hline $\mathrm{Cs}$ & 3.8939 & 137.035999048 & $137.035999046(27)$ & Parker $^{[4]}(2018)$ \\
\hline $\mathrm{Rb}$ & 4.1771 & 137.035999050 & $137.035999037(91)$ & Bouchendira $^{[6]}(2011)$ \\
\hline $\mathrm{H}$ & 13.5984 & 137.035999114 & $137.035999084(21)$ & Codata $(2020)^{(2017)}$ \\
\hline $\mathrm{He}$ & 24.5873 & 137.035999188 & $137.035999180(330)$ & Sun $^{[7]}(2017)$ \\
\hline $\mathrm{Rb}(2)$ & 27.2895 & 137.035999206 & $137.035999206(11)$ & Morel $^{[5]}(2017)$ \\
\hline $\mathrm{He}(2)$ & 54.417 & 137.035999390 & $137.035999550(640)$ & Smiciklas $^{[8]}(2010)$ \\
\hline
\end{tabular}

Notice: $\mathrm{He}(2)$ is not shown figure 2

Acknowledgement:

I would like to thank Norbert Dahmen and Andrea Ehrmann for profound discussions and John Wilcoxen who encouraged me to continue the research 20 years ago.

Data available statement:

Data available on request from the authors 
Literature

(1) Higgs Mechanism: A. Djouadi, arXivhep-ph / 0503172

(2) Preprint Manfred Geilhaupt, Research Square

2.1 Derivation of the FSC

https://www.researchsquare.com/article/rs-524770/v3

2.2 Explanation of the deviation between Morel and Parker results https://www.researchsquare.com/article/rs-579464/v1

(3) Albert Einstein: Grundzüge der Relativitätstheorie Vieweg \&Sohn,Braunschweig, 1969

(4) R.H. Parker (2018): Measurement of the fine-structure constant as a test of the Standard Model, Atomic Physics, Science 13 Apr 2018: Vol. 360, Issue 6385, pp. 191-195, (1/alpha=137.035999046(27) (Cs), 1 lonization energy)

(5) Morel, L., Yao, Z., Cladé, P. et al. Determination of the fine-structure constant with an accuracy of 81 parts per trillion. Nature 588, 61-65 (2020). https://doi.org/10.1038/ s41586-020-2964-7, (1/alpha=137.035999206(11) (Rb), 2 lonization energy)

(6) Rym Bouchendira, Pierre Cladé, Saïda Guellati-Khélifa, François Nez, and François Biraben, Phys. Rev. Lett. 106, 080801 - Published 24 February 2011 ( $\alpha-1=137.035999037(91)(R b), 1$. Ionization energy)

(7) Y. R. Sun, and S.-M. Hu, Precision Spectroscopy of Atomic Helium (2017 He 137.035999180(330)), 2020. Published by Oxford University Press (http:// creativecommons.org/licenses/by/4.0/)

(8) A DETERMINATION OF THE FINE STRUCTURE CONSTANT USING PRECISION MEASUREMENTS OF HELIUM FINE STRUCTURE Marc Smiciklas, B.S., M.S. UNIVERSITY OF NORTH TEXAS August 2010 He: $\alpha-1=137.035999$ 550(640). 